\title{
Surgical and Functional Outcome of Pre-Peritoneal Repair of Inguinal Hernia in Cirrhotic Patient with Mild to Moderate Ascites
}

Hussein Elgohary ${ }^{1 *}$, Ahmed M Nawar ${ }^{1}$, Ahmed Zidan ${ }^{1}$, Ayman M Abdelmofeed ${ }^{1}$, Taher H Elwan ${ }^{1}$, Mohamed I Abourizk ${ }^{1}$ and Ahmed M Hussein ${ }^{2}$

${ }^{1}$ Department of General Surgery, Benha University, Egypt

${ }^{2}$ Department of Internal Medicine, Benha University, Egypt

"Corresponding author: Hussein Elgohary, Department of General Surgery, Faculty of Medicine, Benha University, Egypt, Tel: 201224983326; E-mail: dr_elgohary1996@yahoo.com

Received date: December 13, 2018; Accepted date: December 20, 2018; Published date: December 27, 2018

Copyright: ( 2018 Elgohary H, et al. This is an open-access article distributed under the terms of the Creative Commons Attribution License, which permits unrestricted use, distribution and reproduction in any medium, provided the original author and source are credited.

\begin{abstract}
Objective: To evaluate the outcome of pre-peritoneal repair of an inguinal hernia on both liver functions and quality of life in cirrhotic patients with ascites.

Background: Patient with liver cirrhosis and ascites exhibit peritoneal distension and so increased the incidence of herniation through weakest points in the anterior abdominal wall. Although the incidence of an inguinal hernia in the patient with liver cirrhosis has not been well documented, it is expected to be higher than the general population.

Patient and Methods: The study included 35 cirrhotic patients with mild to moderate ascites. All patients were treated by large prolene mesh patch through pre-peritoneal approach using single transverse supra-pubic incision. Unilateral cases were treated prophylactically for the non-diseased side. All cases were treated on an elective basis. During the $1^{\text {st }} 3$ months post-operative, we re-assess liver functions and compare it with preoperative results, quality of life was tested by the end of $6^{\text {th }}$ month postoperative and evaluations of recurrence is done by the end of the follow-up period.
\end{abstract}

Results: All patients passed a smooth uneventful operative and immediate postoperative course. All patients show no postoperative mortality in the $1^{\text {st }}$ month postoperative. All patients show no post-operative recurrence during follow up period.

Conclusion: Preperitoneal approach for an inguinal hernia in a cirrhotic patient with ascites is safe on liver functions and effective in preventing recurrence.

Keywords Cirrhotic patient; Ascites; Inguinal hernia; Pre-peritoneal repair; Outcome

\section{Introduction}

The prevalence of chronic liver disease and cirrhosis has continued to rise in recent years, especially with the epidemic of viral hepatitis, alcohol abuse, and obesity. Patients with liver cirrhosis and ascites exhibit peritoneal distension and frequently have subsequent herniation of the weakest structures in the abdominal wall [1]. Although the incidence of an inguinal hernia in patients with liver cirrhosis has not been well documented, it is expected to be higher than the general population [2]. Elective abdominal procedures have traditionally been widely discouraged because of the high mortality, risk of postoperative liver decompensation and poor wound healing. However, the watchful waiting approach, on the other hand, may result in emergency surgery which is associated with probably even higher morbidity and mortality for this vulnerable group of patients [3,4]. To assess the utility of surgical repair of a groin hernia in a patient with ascites, the surgeon must weigh the risk of perioperative complication, recurrence and ascetic leak relative to the likelihood of complication from an untreated hernia in a patient who has a poor medical risk [5].
In today's scenario, the Lichtenstein technique has achieved marquee status as the procedure of choice for open repairs. It is a tensionless repair, easy to learn and perform, with very low recurrence rates. However, patients undergoing hernioplasty by Lichtenstein procedure can have wound complaints and chronic groin pain which is often underreported. These problems can be avoided by placing the mesh in the pre-peritoneal plane [6].

\section{Patient and Methods}

The current prospective study was conducted at the General Surgery Department, Benha University Hospital, after obtaining approval from the local ethical committee and after fully informed written consent signed by the patients. This study was carried out on 35 consecutive cirrhotic patients with ascites complaining of an inguinal hernia since May 2013 till September 2018. Out of the thirty-five patients, fifteen patients had a bilateral hernia and the other twenty patients had a unilateral hernia, in the unilateral cases the patient was treated prophylactically for the non-diseased side. Twenty-three patients had a de novo hernia and twelve patients had recurrent hernias

All patients underwent clinical examination including the collection of demographic data and past medical history. All patients underwent laboratory (Complete blood count, Blood sugar, Renal function tests, full Liver function tests, alpha-fetoprotein and PCR for HBV, HCV), 
radiological workup (pelvi-abdominal ultrasound and tri-phasic CT for any suspicious liver lesion found in the ultrasonography) and upper GIT endoscopy. The operation is done after maximum control of ascites and improvement of their coagulation profile.

Inclusion criteria: Cirrhotic patients with controllable ascites with unilateral, bilateral or an even recurrent inguinal hernia.

Exclusion criteria: Liver cirrhosis with uncontrollable or tense ascites, hyper-splenisim, hepato-renal syndrome, primary liver malignancy, active viral hepatitis, previous lower midline incision or Pfannenstiel incision, concomitant hernia as umbilical or epigastric and any other co-morbidity making the patient unfit for surgery.

\section{Surgical procedure}

All operations were done under spinal anesthesia. The operation was done in the supine position using single supra-pubic transverse skin incision about $15 \mathrm{~cm}$ length. Anterior rectus sheath is incised transversally, and flap of the anterior rectus sheath is elevated up and down in order to expose rectus muscle, which in turn retracted laterally, exposing pre-peritoneal space. Careful dissections of hernia sac from the deep inguinal ring is done separating it from vas deference and spermatic vessel and returning it back to the abdomen (Figure 1A), the sac is either ligated or cut or lift without intervention according to its size. In the unilateral cases, the peritoneal sac is dissected medially with capitalization of the vas deference and spermatic vessel as much as possible (at least $10 \mathrm{~cm}$ ). Care is taken to avoid injury of the peritoneum, which if occurs closure is done using absorbable suture.

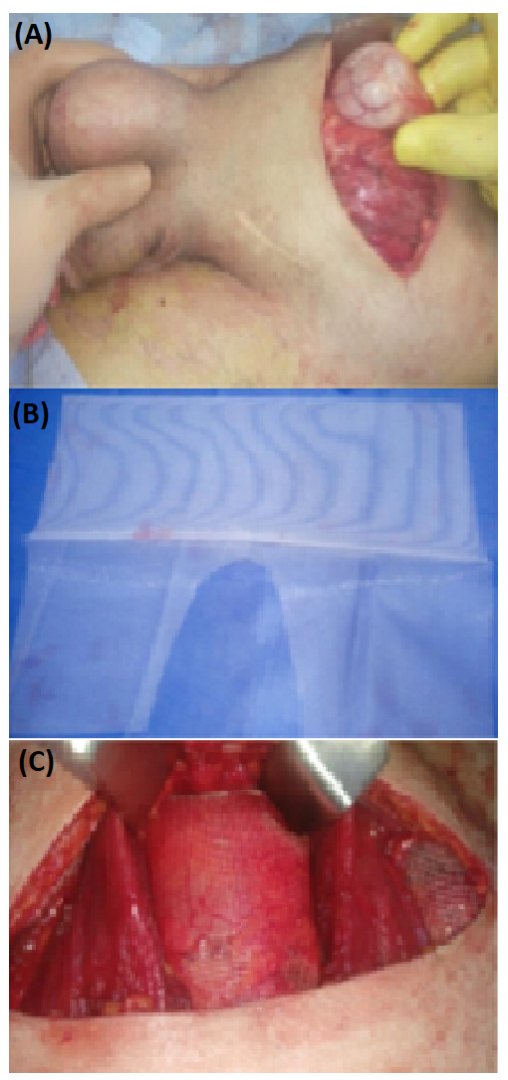

Figure 1: (A) Hernia sac containing asceticfluid; (B) Mesh design; (C) Placement of the mesh in the pre-peritoneal space.
In an indirect hernia, the deep inguinal ring is narrowed from inside by non-absorbable prolene suture while in the direct type of a hernia the redundant fascia transversalis is stretched and fixed to the bone. The mesh is inserted. The used prolene mesh is designed to be like pantalon (Figure 1B) with the two limbs were inserted in the back of both inguinal regions overriding urinary bladder and supporting the back of Hesselbach's triangle, deep inguinal ring, and femoral ring. The upper border of the mesh runs transversally reaching above the level of arcuate line and laterally up to $10 \mathrm{~cm}$ lateral to the deep inguinal ring. The mesh is fixed to the narrowed deep ring bilaterally (site of injury of the inferior epigastric vessel) and to the arcuate line above (Figure 1C). Suction drain $18 \mathrm{~F}$ is inserted over the mesh. Both recti were approximated over the mesh, closure is done in layers.

\section{Post-operative care}

All patients have restricted to the protein intake of $1.5 \mathrm{gm} / \mathrm{kg} / \mathrm{day}$ and salt restriction to $2 \mathrm{gm}$. All patients received postoperative intravenous antibiotics ( $4^{\text {th }}$ generation cephalosporin $1 \mathrm{gm} / 12 \mathrm{~h}$ ) for $1^{\text {st }} 5$ days then continue on an oral antibiotic, adequate postoperative analgesia using paracetamol $1 \mathrm{gm}$ infusion every 8 hours (opioids and other NSAIDs were restricted), K-sparing diuretic and liver supporting drugs. Lactulose to maintain two bowel motion per day. Some patients receive human albumin infusion. Discharge after $5 \pm 2$ days. The drain is usually removed in the $1^{\text {st }}$ week. Recovery time is usually around 2 weeks.

\section{Study outcome}

\section{Surgical outcome:}

- Intraoperative (IO) collected data included operative time, IO blood loss, and frequency of IO complications

- Postoperative (PO) data included, PO hospital stay, the frequency of $\mathrm{PO}$ complication and time needed for mesh incorporation

- Postoperative (PO) follow-up extending for 24 months for the frequency of recurrence

Functional outcome: It was assessed and compared versus preoperative evaluation for-

- The effect of this type of repair on liver functions by comparing the pre-operative results of the Child-Turcotte-Pugh (CTP) score and Model of End-Stage Liver Disease (MELD) score with the postoperative results at 1 and 3 months postoperative

- Evaluation of the effect of this type of repair on the improvement of the quality of life QOL by comparing the pre-operative results of the two main components of the Short Form (36) Health Survey (SF-36); (physical component summary and mental component summary) with the post-operative results 3 and 6 months postoperative

\section{Statistical Analysis}

The program used was SPSS version 20. Quantitative data were analyzed using mean and standard deviation, while frequency and percentage were used with qualitative data. Paired t-test and Wilcoxon test were used to compare means of different variables among the same group. Differences were considered significant at $\mathrm{p} \leq 0.05$.

\section{Results}

The study included 35 patients; 33 males and 2 females with a mean age of $51 \pm 3.6$ years (range: $42-65$ years). The mean body mass index was $24.5 \pm 2.6 \mathrm{~kg} / \mathrm{m}^{2}$. The detailed characters of the studied group are shown in (Table 1). 


\begin{tabular}{|l|l|l|}
\hline & Number & Percentage \\
\hline History & 7 & $20 \%$ \\
\hline Encephalopathy & 6 & $17.14 \%$ \\
\hline Hematemesis & 6 & $42.85 \%$ \\
\hline Melena & 15 & $60 \%$ \\
\hline Bilharziasis & 21 & \\
\hline
\end{tabular}

\section{Previous history of hernia repair}

\begin{tabular}{|l|l|l|}
\hline Umbilical & 11 & $31.42 \%$ \\
\hline $\begin{array}{l}\text { Unilateral inguinal (6 cases were recurrent and } 3 \\
\text { cases had a de novo hernia on the other side) }\end{array}$ & 9 & $25.71 \%$ \\
\hline $\begin{array}{l}\text { Bilateral inguinal (4 cases were unilateral } \\
\text { recurrent and 2 cases were bilateral recurrent) }\end{array}$ & 6 & $17.14 \%$ \\
\hline
\end{tabular}

\section{Concomitant Disease}

\begin{tabular}{|l|l|l|}
\hline None & 14 & $40 \%$ \\
\hline Diabetes Mellitus & 8 & $22.85 \%$ \\
\hline Cardiac disease & 3 & $8.57 \%$ \\
\hline COPD & 11 & $31.42 \%$ \\
\hline Benign prostatic hypertrophy & 17 & $48.57 \%$ \\
\hline Cerebro-vascular accident & 1 & $2.85 \%$ \\
\hline The side of a hernia & \multicolumn{2}{|l|}{} \\
\hline Right & 12 & $34.28 \%$ \\
\hline Left & 8 & $22.85 \%$ \\
\hline Bilateral & 15 & $42.85 \%$ \\
\hline Etiology of cirrhosis & \multicolumn{2}{|l}{} \\
\hline Hepatitis C & 32 & $91.42 \%$ \\
\hline Hepatitis B & 3 & $8.57 \%$ \\
\hline Combined hepatitis C and alcohol abuse & 1 & $2.85 \%$ \\
\hline
\end{tabular}

Type of a hernia (50 hernias)

\begin{tabular}{|l|l|l|}
\hline Indirect & 38 & $76 \%$ \\
\hline Direct & 12 & $24 \%$ \\
\hline
\end{tabular}

\section{Amount of ascites}

\begin{tabular}{|l|l|l|}
\hline Mild & 14 & $40 \%$ \\
\hline Moderate & 21 & $60 \%$ \\
\hline
\end{tabular}

\section{Child-Pugh classification}

\begin{tabular}{|l|l|l|}
\hline Class-B & 28 & $80 \%$ \\
\hline Class-C & 7 & $20 \%$ \\
\hline
\end{tabular}

Table 1: Shows the detailed characteristics of the studied group.
All patients passed a smooth uneventful operative and immediate postoperative course. All surgeries were conducted through a mean operative time of $90 \pm 15$ min which is suitable for bilateral repair and average blood loss of $180 \pm 70 \mathrm{ml}$ with no need for blood or platelet transfusion. Meticulous dissection for vas deferens and spermatic vessels from hernia sac makes their injury is easily avoidable. However, lateral retraction of rectus muscle cause injury of inferior epigastric vessels in 5 cases which was treated by ligation. The peritoneal tear occurs in 6 cases, all of them were recurrent; this leads to leakage of ascites with an average amount of $150 \pm 50 \mathrm{ml}$. All cases with moderate ascites after maximum control of their ascites receive routinely postoperative human albumin infusion together with diuretics. The hospital discharge is usually after 2 days that may extend to 7 days if there is a complication (Table 2).

\begin{tabular}{|l|l|}
\hline Optime & $90 \pm 15 \mathrm{~min}$ \\
\hline Op blood loss & $180 \pm 70 \mathrm{ml}$ \\
\hline Need for blood transfusion & ------ \\
\hline Need for platelet transfusion & ------ \\
\hline Injury of the vas or spermatic vessel & ------- \\
\hline Injury to inferior epigastric vessels & 5 cases \\
\hline Peritoneal tear and ascites leak & 6 cases \\
\hline Amount of ascites lost intra-operative & $150 \pm 50 \mathrm{ml}$ \\
\hline Post-operative human albumin infusion & 20 case \\
\hline Hospital stay & $2: 7$ days \\
\hline
\end{tabular}

Table 2: Shows operative and postoperative data.

After the operation, all patients (100\%) show complete recovery with no mortality or major morbidity in the early postoperative period. However minor complications occur like the development of wound seroma in four obese cases $(11.42 \%)$ treated by drainage, three diabetic cases $(8.57 \%)$ develop superficial wound infection treated by drainage and prolongation of antibiotic therapy and two cases (5.71\%) develops scrotal hematoma that is treated conservatively. Edema and hardness of the cord occur only in four of the recurrent cases (11.42\%) that are generally not annoying the patients and pass off spontaneously within $1^{\text {st }}$ two months post-operative. The majority of cases develop lower abdominal tightness and strapping sensation in up to (71.42\%) of the cases, this is due to the large sized mesh patch used, and also the sustained high intra-abdominal pressure makes this type of sensation obvious in a patient with moderate ascites.

However, this sense of tightness' does not affects the patients' daily working activity or causing chronic lower abdominal pain and relived spontaneously in the $1^{\text {st }}$ month postoperative. There is no chronic groin pain as in the ordinary repair of an inguinal hernia. Two patients (5.71\%) with CTP score class C develops hepatic pre-coma and they respond rapidly to conventional measures in the intensive care unit. All operations were done under spinal anesthesia without complications except for the development of post spinal headache in three cases $(8.57 \%)$ treated by paracetamol and caffeine-containing drugs. 
Citation: Elgohary H, Nawar AM, Zidan A, Abdelmofeed AM, Elwan TH, et al. (2018) Surgical and Functional Outcome of Pre-Peritoneal Repair of Inguinal Hernia in Cirrhotic Patient with Mild to Moderate Ascites. Surgery Curr Res 8: 317. doi:10.4172/2161-1076.1000317

Page 4 of 6

Two cases (5.71\%) develop urine retention that necessitates catheterization. There is no reported cases of deep infection reaching the mesh or necessitates mesh removal. Repeated superficial ultrasonography is performed every two months to show mesh incorporation into tissue. Twenty patients (57.14\%) shows complete mesh incorporation within the $1^{\text {st }} 4$ months post-operative and 11 patient $(31.42 \%)$ develops mesh incorporation by the end of $6^{\text {th }}$ month, while the remaining $4(11.42 \%)$ patients show complete mesh incorporation by the end of 8 th months post-operative. After the end of the follow-up period, there is no reported case of recurrence neither in the treated diseased side nor in the side which is treated prophylactically. However, there are five cases (14.28\%) develops an umbilical hernia about 11 to 17 months post-operative, two of them undergo repair (Table 3).

\begin{tabular}{|c|c|}
\hline \multicolumn{2}{|l|}{ Complication } \\
\hline Wound seroma & 4 cases $(11.42)$ \\
\hline Wound infection & 3 cases $(8.57)$ \\
\hline Scrotal hematoma & 2 cases $(5.71)$ \\
\hline Lymphedema of the cord & 4 cases $(11.42)$ \\
\hline Lower abdominal tightness & 25 cases $(71.42)$ \\
\hline Chronic groin pain & ------- \\
\hline Hepatic pre-coma & 2 cases $(5.71)$ \\
\hline Hepatic coma & ------ \\
\hline ICU admission & 2 cases $(5.71)$ \\
\hline \multicolumn{2}{|l|}{ Anesthetic complication } \\
\hline Post spinal headache & 3 cases $(8.57)$ \\
\hline Urine retention & 2 cases $(5.71)$ \\
\hline Development of an umbilical hernia & 5 cases $(14.28)$ \\
\hline Mesh infection & ----- \\
\hline Recurrence (During the follow-up period) & ----- \\
\hline
\end{tabular}

Table 3: Shows post-operative complication.

The most commonly used scoring systems for the evaluation of liver functions and predicting morbidity and mortality among cirrhotic patients are Child-Turcotte-Pugh (CTP) and Model for End-Stage Liver Disease (MELD) score. In this study, we compare the mean preoperative CPT and MELD score for every patient with the mean postoperative score after the $1^{\text {st }}$ month and $6^{\text {th }}$ month.
There is a mild increase in both CTP and MELD score after the $1^{\text {st }}$ month post-operative while these results return again near the preoperative values by the $3^{\text {rd }}$ month. There is a mild drop in albumin level and increase in bilirubin level while INR and serum creatinine shows no significant change through the post-operative period. The amount of ascites also shows little increase in its amount which is gradual returns to preoperative values concomitant with the improvement in serum albumin level. Only two patients develop hepatic encephalopathy grade one that is corrected rapidly and not repeated during the follow -up period (Tables 4 and 5).

\begin{tabular}{|l|l|l|l|}
\hline CTP score & \multicolumn{3}{|l|}{ The mean results for 35 patients } \\
\hline $\begin{array}{l}\text { Laboratory and clinical } \\
\text { parameters }\end{array}$ & Pre-operative & $\begin{array}{l}\text { 1st month } \\
\text { post- } \\
\text { operative }\end{array}$ & $\begin{array}{l}\text { 3rd month post- } \\
\text { operative }\end{array}$ \\
\hline Albumin, g/dl & $2.84 \pm 0.61$ & $2.21 \pm 0.30^{\wedge}$ & $2.77 \pm 0.71 \$$ \\
\hline Bilirubin, mg/dl & $1.60 \pm 1.07$ & $1.83 \pm 1.29^{\wedge}$ & $1.68 \pm 1.14 \$$ \\
\hline INR & $1.41 \pm 0.32$ & $1.51 \pm 0.30^{\wedge}$ & $1.39 \pm 0.36 \$$ \\
\hline Hepatic encephalopathy & $7(20.0)$ & $2(5.7)$ & $0(0.0)$ \\
\hline Ascites, ml & $1800.6 \pm 399.4$ & $2000 \pm 500^{\wedge}$ & $1772.9 \pm 379.3 \$$ \\
\hline Total score & $8.11 \pm 0.71$ & $8.9 \pm 0.6^{\wedge}$ & $8.23 \pm 1.06 \$$ \\
\hline$\wedge=$ sig and pre-op; \$=sig and $1^{\text {st }}$ month post-op & \\
\hline
\end{tabular}

Table 4: Shows the CTP score.

\begin{tabular}{|l|l|l|l|}
\hline MELD score & \multicolumn{3}{|l|}{ The mean results for 35 patients } \\
\hline Laboratory parameters & Pre-operative & $\begin{array}{l}1^{\text {st }} \text { month post- } \\
\text { operative }\end{array}$ & $\begin{array}{l}3^{\text {rd }} \text { month post- } \\
\text { operative }\end{array}$ \\
\hline Creatinine, mg/dl & $0.51 \pm 0.39$ & $0.7 \pm 0.59^{\wedge}$ & $0.57 \pm 0.44 \$$ \\
\hline Bilirubin, mg/dl & $1.63 \pm 1.1$ & $1.8 \pm 1.3^{\wedge}$ & $1.66 \pm 1.09 \$$ \\
\hline INR & $1.4 \pm 0.33$ & $1.5 \pm 0.31^{\wedge}$ & $1.38 \pm 0.34 \$$ \\
\hline Total score & $11.35 \pm 1.09$ & $12.78 \pm 0.61^{\wedge}$ & $11.30 \pm 1.06 \$$ \\
\hline$\wedge=$ sig and pre-op; $\$=$ sig and $1^{\text {st }}$ month post-op & \\
\hline
\end{tabular}

Table 5: Shows the MELD score.

The two indexes of the SF-36 survey (MCS) and (PCS) scores are significantly increased as compared with preoperative values. The increases in the values for the (PCS) are significantly higher than the values for the (MCS) (92.34\% improvement in PCS compared to $47.63 \%$ in MCS). This gives a clear indication of the improvement in the quality of life in patients undergoing this type of repair (Table 6).

\begin{tabular}{|c|c|c|c|c|}
\hline \multirow{2}{*}{ Component of SF-36 } & \multicolumn{4}{|c|}{ The mean results for 35 patients } \\
\hline & Pre-operative & 3 months postoperative & 6 months postoperative & Improvement \% \\
\hline Mental Component Summary (MCS) & $207.69 \pm 21.16$ & $268.03 \pm 16.08^{\wedge}$ & $304.94 \pm 15.8^{\wedge} \$$ & $47.63 \pm 8.76$ \\
\hline Physical Component Summary (PCS) & $174.8 \pm 32.12$ & $236.43 \pm 52.23^{\wedge}$ & $329.23 \pm 29.31^{\wedge} \$$ & $92.34 \pm 26.12$ \\
\hline
\end{tabular}

Table 6: Shows the SF-36 survey. 


\section{Discussion}

Patients with liver cirrhosis and ascites had an increase in the incidence of hernias in general. It greatly complicates the decisionmaking process necessary to provide optimal care [7]. The problem in these patients is the chronic increased intra-abdominal pressure as well as muscular wasting, a consequence of malnutrition, are thought to be the main predisposing factors to hernia development. Groin hernias are seen less frequently in cirrhotic patients than umbilical hernias. However, complications are more frequently seen with groin hernias [8]. Elective repair for hernias has traditionally been widely discouraged because of the high mortality, risk of postoperative liver decompensation and poor wound healing. However remaining under observation, on the other hand, may result in emergency surgery which is associated with higher morbidity and mortality for this vulnerable group of patients [3].

Mesh repair has become a standard surgical technique in hernia repair. However, in chronic liver disease patients use of mesh is considered a relative contraindication by some because of poor tissue incorporation of mesh and risk of infection. On the other hand, simple suture repair may result in a higher recurrence rate. In a randomized trial, authors found that in patients with cirrhosis, recurrence of a hernia was lower after mesh repair (2.7\%) compared to suture repair (14.2\%). However, mesh repairs were more likely to become infected (16.2\% vs. $8.5 \%)$ but the results were statistically insignificant [3].

Lichtenstein hernioplasty represents the commonest type of repair for an inguinal hernia. However, it still not protecting a femoral opening or obturator opening on the same side and the other side is inaccessible. Also, it remains under the stress of continuous increased intra-abdominal pressure from its posterior aspect and lack of support from its anterior aspect makes it reliable for recurrence [9].

In the present study, the use of a pre-peritoneal approach for inguinal hernia repair using a large mesh patch isn't a new technique. Its use in cirrhotic patients with ascites, who have continuous high intra-abdominal pressure, very week anterior abdominal wall musculature and have a high incidence of developing unilateral or bilateral or even recurrent hernia, is very useful. The supra-pubic transverse skin incision provides access for bilateral repair of groin hernias using the same incision. The use of large mesh patch protects against the development of not only both direct and indirect inguinal hernia but also femoral and obturator hernias. The position of the mesh in the pre-peritoneal space behind the full thickness of the anterior abdominal wall gives great support against the high intraabdominal pressure. Mesh fixation to the back of the narrowed internal ring and to the arcuate line above prevents sliding of the sac again in front of the mesh, so it is considered a form of reconstruction of the lower anterior abdominal wall in this vulnerable group of patients.

Hurst et al reported that life-threatening complications from inguinal hernia repair in patients with cirrhosis and ascites are uncommon. Morbidity and long-term mortality rates in these patients are due to the progression of the underlying liver disease. Hurst et al reviewed 18 patients with cirrhosis accompanied by ascites and groin hernia (20 inguinal and one femoral), no major and four minor postoperative complications occurred, and there were no cases of perioperative death or ascites leakage [7].
Rubik Ray et al., compare trans-inguinal pre-peritoneal approach with Lichtenstein hernioplasty and shows that the Lichtenstein group had increased incidence of the wound and scrotal collection in the immediate post-operative period, as well as wound induration and chronic pain in long-term follow-up. Polypropylene mesh can cause light microscopic and ultra-structural changes in the peripheral nerve due to myelin degeneration, endoneurial and perineurial edema, fibrosis, axonal loss [10], and can cause chronic groin pain by entrapment of the nerve in scar tissue. Wound seroma and induration can be caused by foreign body reaction to mesh [11]. Chronic pain and wound complaints delay recovery and can significantly affect a patient's daily lifestyle [6]. All these complications are avoided by placing the mesh in the pre-peritoneal plane.

In the present study, all patients (100\%) show complete recovery with no mortality or major morbidity in the early postoperative period. However minor complications like wound seroma (11.42\%), scrotal hematoma $(5.71 \%)$, and superficial wound infections $(8.57 \%)$ were treated conservatively. There is no chronic groin pain, however, the majority of cases $(71.42 \%)$ develop lower abdominal tightness and strapping sensation that does not affect the patients' daily working activity and relived spontaneously in the $1^{\text {st }}$ month postoperative.

The pre-peritoneal space is full of adipose tissue, connective tissue and membranous tissue thus facilitating mesh incorporation [12], in the present study repeated superficial ultrasonography to the lower anterior abdominal wall reveals that up to 20 patients (57.14\%) shows complete mesh incorporation within the $1^{\text {st }} 4$ months post-operative and 11 patient $(31.42 \%)$ develops mesh incorporation by the end of $6^{\text {th }}$ month, while the remaining $4(11.42 \%)$ patients shows complete mesh incorporation by the end of 8 th months post-operative.

Patients with cirrhosis have a significant risk of adverse outcome after abdominal wall hernia repair compared to non-cirrhotic patients. Ideally, patients with cirrhosis should undergo elective hernia repair after medical optimization [13]. In the present study, patients had all responsive ascites and had been treated continuously at the department of internal medicine for control of ascites with appropriate sodium restriction and diuretic therapy and improvement of coagulation profile and general condition before elective hernia repairs. There was no outstanding change between the Child-Pugh score or MELD score just before surgery and three months postoperative.

In recent times, quality of life has become a very important aspect of patient care. Patti et al used the Short Form-36 (SF-36) questionnaire to evaluate QOL in patients with cirrhosis undergoing inguinal hernia repair to identify optimal management of symptomatic inguinal hernia in patients with cirrhosis. All eight SF-36 domains and the mental component summary and physical component summary scores improved remarkably after hernia repair, especially in patients in Child's class C and/or those with refractory ascites [14]. Lawson et al, in a randomized clinical trial of patients undergoing repair of an inguinal hernia, found significant improvement in the quality of life, also assessed by the SF-36 scores between the pre-operative and postoperative [15]. Young Hoe Hur et al reported that inguinal hernia repair is a safe procedure for treatment of symptomatic inguinal hernia in patients with cirrhosis. The improvement in QOL represents a clearcut indication for elective hernia repair [16]. 
Citation: Elgohary H, Nawar AM, Zidan A, Abdelmofeed AM, Elwan TH, et al. (2018) Surgical and Functional Outcome of Pre-Peritoneal Repair of Inguinal Hernia in Cirrhotic Patient with Mild to Moderate Ascites. Surgery Curr Res 8: 317. doi:10.4172/2161-1076.1000317

Page 6 of 6

In the present study, the same results were also recorded as the two indexes of the SF-36 survey (MCS) and (PCS) scores are significantly increased as compared with pre-operative values with a marked improvement (92.34\%) in the (PCS) than(47.63\%)in the (MCS).

Hurst RD et al. shows one recurrence (8\%) over an average25month follow-up period suggests that most repairs can remain intact for the life expectancy of the cirrhotic with ascites [7]. Oh HK et al., shows three recurrences $(2.3 \%)$ out of 129 patients with liver cirrhosis underwent inguinal hernia repair with the slandered Mc Vay procedure over a 10-year period (median follow up 22.9 months) [4]. In the present study, there are no reported cases of recurrence or development of contralateral side hernia during the follow- up period.

\section{Conclusion}

Elective repair of an inguinal hernia in a cirrhotic patient with ascites greatly improve quality of life and is truly indicated. Proper preoperative optimization of general condition especially controls of ascites and coagulation profile was the cornerstone for a smooth postoperative outcome. Pre-peritoneal approach greatly meets the needs of cirrhotic patients with ascites in the repair of their groin hernias. The use of large mesh patch through single supra-pubic incision allows access for repair of both sides or prophylactic repair of another side also de novo approach for a recurrent hernia previously treated by the inguinal approach. Presence of the mesh in the pre-peritoneal space gives it more support and resistance to the continuously high intraabdominal pressure with good mesh incorporation with no reported cases of mesh infection or hernia recurrence in the present study. From the previously mentioned, we conclude that the pre-peritoneal approach is a safe procedure for the management of an inguinal hernia in a cirrhotic patient with ascites within reasonable operative time and minimal postoperative morbidities.

\section{References}

1. Park JK, Lee SH, Yoon WJ, Lee JK, Park SC, et al. (2007) Evaluation of hernia repair operation in Child-Turcotte-Pugh class $\mathrm{C}$ cirrhosis and refractory ascites. J Gastroenterol Hepatol 22: 377-382.

2. Belghiti J, Durand F (1997) Abdominal wall hernias in the setting of cirrhosis. Semin Liver Dis 17: 219-226.
3. FSilva FD, Andraus W, Pinheiro RS, Arantes-Junior RM, Lemes MP, et al. (2012) Abdominal and inguinal hernia in cirrhotic patients: What's the best approach? Arq Bras Cir Dig 25: 52-55.

4. Oh HK, Kim H, Ryoo S, Choe EK, Park KJ (2011) Inguinal hernia repair in patients with cirrhosis is not associated with an increased risk of complications and recurrence. World J Surg 35: 1229-1233.

5. de Goede B, Klitsie PJ, Lange JF, Metselaar HJ, Kazemier G (2012) Morbidity and mortality related to non-hepatic surgery in patients with liver cirrhosis: A systematic review. Best Pract Res Clin Gastroenterol 26: 47-59.

6. Koning GG, Koole D, de Jongh MAC, de Schipper JP, Verhofstad MHJ, et al. (2011) The transinguinal preperitoneal hernia correction vs. Lichtenstein technique; is TIPP top? Hernia 15: 19-22.

7. Hurst RD, Butler BN, Soybel DI, Wright HK (1992) Management of groin hernias in patients with ascites. Ann Surg 216: 696-700.

8. Leonetti JP, Aranha GV, Wilkinson WA, Stanley M, Greenlee HB (1984) Umbilical herniorrhaphy in cirrhotic patients. Arch Surg 119: 442-445.

9. Koc M, Aslar AK, Yoldas O, Ertan T (2004) Comparison of quality of life outcomes of stoppa vs. bilateral Lichtenstein procedure. Hernia 8: 53-55.

10. Seher D, Ilknur K, Evirgen O, Birsen O, Tuzuner A, et al. (2006) The effect of polypropylene mesh on ilioinguinal nerve in open mesh repair of groin hernia. J Surg Res 131: 175-181.

11. Vironen J, Nieminen J, Eklund A, Paavolainen P (2006) Randomized clinical trial of Lichtenstein patch or Prolene Hernia System for inguinal hernia repair. Br J Surg 93: 33-39.

12. Mirilas P, Colborn GL, McClusky DA, Skandalakis LJ, Skandalakis PN, et al. (2005) The history of anatomy and surgery of the preperitoneal space. Arch Surg 140: 90-94.

13. Carbonell AM, Wolfe LG, DeMaria EJ (2005) Poor outcomes in cirrhosisassociated hernia repair: A nationwide cohort study of 32,033 patients. Hernia 9: 353-357.

14. Patti R, Almasio PL, Buscemi S, Fama F, Craxi A, et al. (2008) Inguinal hernioplasty improves the quality of life in patients with cirrhosis. Am J Surg 196: 373-378.

15. Lawson EH, Benjamin E, Busuttil RW, Hiatt JR (2009) Groin herniorrhaphy in patients with cirrhosis and after liver transplantation. Am Surg 75: 962-965.

16. Hur YH, Kim JC, Kim DY, Kim SK, Park CY (2011) Inguinal hernia repair in patients with liver cirrhosis accompanied by ascites. J Korean Surg Soc 80: 420-425. 